\title{
Capital humano e ingresos en la manufactura de Tijuana y Mexicali, 1994-2001
}

\author{
Claudio Cabrera, Alejandro Mungaray, \\ Rogelio Varela y Emilio Hernández*
}

\begin{abstract}
Resumen. El presente trabajo analiza los ingresos de los empleados de las manufacturas de Tijuana y Mexicali en función de los niveles de escolaridad y la experiencia laboral a partir de un modelo econométrico de capital humano. Con información proveniente de la Encuesta Nacional de Empleo Urbano (ENEU), se integró una base de datos de panel representativa con una muestra de 184 observaciones para Tijuana entre 1994 y 2001 (8 años y 23 ramas) y de 112 para Mexicali entre 1998 y 2001 (4 años y 28 ramas). El hecho de que la escolaridad sea más importante que la experiencia laboral en la determinación del nivel de ingresos de los trabajadores de la industria manufacturera para Tijuana y Mexicali, significa que en el mercado de trabajo local el nivel de educación es un criterio fundamental de la negociación salarial.

Palabras clave: educación, escolaridad, experiencia, ingresos, manufactura.
\end{abstract}

\begin{abstract}
This work study the income of the Tijuana and Mexicali industrial manufacturing worker as a function of the schooling level and work experience through a human capital econometric model. Information from the National Survey of Urban Employment (ENEU), was used in a representative data base with a sample of 184 observations for Tijuana between 1994 and 2001 (8 years and 23 industrial branches) and 112 observations for Mexicali between 1998 and 2001 (4 years and 28 industrial branches). Schooling was most important than work experience in the income determination of the Tijuana and Mexicali industrial workers. This means that in the local labor market, the educational level is a key factor in the wage negotiation.

Keywords: education, schooling, experience, income, manufacturing.
\end{abstract}

* Cabrera es profesor de tiempo completo en la Escuela de Ingeniería y Negocios de San Quintín de la UABC; Mungaray, Varela y Hernández son profesores de tiempo completo en la FERI-UABC y miembros del Sistema Nacional de Investigadores. 


\section{Introducción}

La fragmentación de la línea de producción para relocalizar procesos productivos donde se pueden minimizar costos es una de las explicaciones de la aglomeración industrial en Baja California, por las externalidades que genera. Esto ha permitido que en el mercado de trabajo local se favorezca la generación de empleo y la oportunidad de aprendizaje empresarial, especializándose en actividades de productos metálicos, maquinaria y equipo y alimentos, bebidas y tabaco.

Esto ha aumentado las expectativas de bienestar social, debido a que la dinámica del empleo significa la posibilidad de acceder a ingresos y con ello a condiciones de desarrollo personal. En este sentido, el propósito de este trabajo es analizar que tanto variables de capital humano como el grado de escolaridad, la experiencia laboral y la especialización del personal ocupado, han sido relevantes en la determinación de los ingresos promedios de los trabajadores de la industria manufacturera de las ciudades de Tijuana y Mexicali, Baja California.

Trabajos cuyas contribuciones son un referente esencial como el de Zepeda y Ghiara (1999), analizan la determinación de los salarios en México desde una perspectiva de capital humano para el periodo 19871993, y encuentran que la dispersión salarial es significativa entre y al interior de las diversas ocupaciones laborales de la industria manufacturera, aun cuando los niveles de escolaridad se homogeneizan. Desde una perspectiva análoga, Cortez (2005) estima una ecuación minceriana para el periodo 1984-2000 e introduce la edad y un conjunto de variables dicotómicas explicativas de los salarios que capturan diferencias interestatales, género, sindicalización, tipo de ocupación y sector de pertenencia. Encuentra que las diferencias salariales interestatales por nivel educativo han sido muy marcadas y estables en el tiempo y concluye que dichas diferencias sólo se han reducido en el caso de los trabajadores que registran niveles de educación superior. Este efecto diferenciado se asocia al proceso de liberalización económica y la exigencia de fuerza de trabajo más especializada en el periodo analizado. Urciaga (2002) estima una ecuación minceriana en donde se especifican los ingresos laborales por hora en función de la 
edad y distintos niveles de escolaridad como primaria, secundaria, preparatoria y superior. Con base en información de la Encuesta Nacional de Ingresos y Gasto de los Hogares Mexicanos correspondiente a 1998, concluye que en México los ingresos dependen en mayor magnitud del grado de escolaridad asociado a la educación superior y en menor medida del nivel medio superior. Mendoza (2002) esboza que la educación formal es el factor más relevante en la determinación del ingreso de los trabajadores de la ciudad de Tijuana y Ciudad Juárez y puntualiza que la experiencia laboral tiene un efecto positivo pero menos significativo que la escolaridad. En esta misma vertiente, Ampudia (2007) encuentra que entre 1987 y 1998 los salarios de los trabajadores de Ciudad Juárez se explican de manera importante por los mayores niveles de educación. El hecho de que muchos jóvenes se incorporen al mercado de trabajo antes de concluir los estudios formales significa que van acumulando experiencia laboral. Por su parte, Burgos (2005) afirma que entre 1994 y 2002 el incremento en la dispersión de los sueldos fue mayor que en la de los salarios, lo que significa que la demanda de trabajo se orientó a favor de los trabajadores con mayor nivel de educación.

Conviene destacar que la gran mayoría de los trabajos realizan estimaciones con base en información de corte transversal y utilizan el método de mínimos cuadrados ordinarios. El trabajo de Barceinas (2001) se caracteriza por efectuar estimaciones con base en un modelo de elección discreta-Probit, concluyendo que la inversión en capital humano mediante la educación puede ser considerada una actividad rentable tanto individual como socialmente. Dentro de esta discusión es muy limitada la literatura que aborda la experiencia mexicana considerando datos de panel. En Huesca (2004) se estima una ecuación de ingresos a partir de una base de datos de panel que contempla los años de 1984, 1989, 1992, 1994, 1996, 1998 y 2000 referentes a información de la Encuesta Nacional de Ingreso y Gasto de los Hogares. En un primer modelo determina que la escolaridad tiene un impacto mayor en los salarios de los jefes de familia que la experiencia laboral, mientras que en el modelo extendido en donde desagrega la escolaridad encuentra que los rendimientos más elevados están vinculados con la formación profesional. 
El presente trabajo parte de la perspectiva de la teoría del capital humano que postula la hipótesis del predominio de los factores de oferta del mercado de trabajo en la explicación de las diferencias salariales. En esta visión el modelo estándar del mercado de trabajo plantea que el salario depende de la productividad laboral, la cual está determinada por la dotación de capital humano, es decir, por el grado de escolaridad y la experiencia laboral (Palacio y Simón, 2002 y 2004). A partir de este enfoque se estiman tres modelos uniecuacionales para el caso de Tijuana y Mexicali considerando las ramas de la industria manufactura. Las variables explicativas de los niveles de ingreso son la escolaridad, la experiencia y su cuadrado. Tanto para el caso de Tijuana como de Mexicali se constata que la escolaridad es un determinante más relevante que la experiencia laboral. Esto sugiere que en ambas ciudades los ingreso promedio de los trabajadores de la industria manufacturera están estrechamente relacionados con los años de escolaridad de los jefes de familia de los hogares. En consecuencia, se puede afirmar que las capacidades y habilidades de la fuerza de trabajo están estrechamente relacionadas con una mayor formación profesional.

El trabajo se divide en cuatro secciones. En la primera se describen aspectos relacionados con el entorno fronterizo de Baja California así como con los ingresos manufactureros y los niveles de escolaridad de Tijuana y Mexicali; en la segunda se especifica un modelo econométrico de capital humano considerando datos de panel con efectos fijos. En la tercera se analizan algunos estadísticos descriptivos de las variables y los resultados de estimación. Finalmente, en la cuarta sección, se plantean algunas conclusiones generales.

\section{Capital humano e ingresos laborales en la industria manufacturera de Baja California}

La aglomeración industrial producida por el establecimiento de un creciente número de empresas en la zona fronteriza de Baja California ha impulsado una creciente especialización en los procesos productivos de actividades industriales manufactureras asociadas al subsector de metal mecánica, maquinaria y equipo y el de alimentos, bebidas y 
tabaco. Entre los principales determinantes de esta concentración se encuentra la vinculación de éstas hacia atrás y hacia delante buscando economías de escala, inducido por la búsqueda de externalidades intra e interindustriales a escala regional. Esto ha generado condiciones de aprendizaje e innovación empresarial con efectos favorables en el empleo y la productividad.

Las ciudades adyacentes a la frontera México-Estados Unidos forman pares binacionales con intensos procesos transfronterizos. Se puede afirmar que el crecimiento económico y poblacional de las ciudades fronterizas mexicanas está relacionado con las crecientes diferencias estructurales entre ambos países, que incentivan los flujos migratorios y la expansión del mercado local de trabajo. Alegría (1992) sostiene que la trasmigración materializa la relación interurbana de cada par de ciudades adyacentes, producto de la contigüidad geográfica de diferentes salarios y precios, que genera una oferta para una demanda de trabajo en un mercado laboral binacional. Para las ciudades fronterizas de Estados Unidos, la trasmigración representa el uso de un recurso localizado, abundante mano de obra con poca paga y bajo potencial de sindicalización y la posibilidad de disminución del salario medio en algunos sectores.

En el contexto fronterizo México-Estados Unidos se desarrollan procesos de innovación y aprendizaje empresarial que fortalecen el desempeño competitivo de la estructura industrial local. De acuerdo con Villavicencio (2002), la innovación implica adquirir conocimiento externo y difundirlo a las unidades productivas y organizativas de la empresa, capitalizar experiencias pasadas de resolución de problemas, crear vínculos estratégicos con instituciones generadoras de conocimiento y mejorar los procesos productivos. La innovación se origina en las redes de cooperación regional enfocadas a la mejora de la gestión técnico-empresarial para competir y generar empleos. A la vez que impulsan el desarrollo de la competitividad, estas redes detectan las necesidades comunes de formación, educación e infraestructura que favorecen el desarrollo empresarial. Las asociaciones y cámaras empresariales también constituyen un importante impulso a las redes de conocimiento, al igual que las asociaciones de productores relacionadas con un producto o proceso en particular (Mungaray, 2001). 
Un enfoque de clusters puede dimensionar el desarrollo empresarial regional con diferentes resultados. En una dimensión horizontal, los agrupamientos de empresas similares produciendo bienes similares, se encuentran localizados unos junto a otros. En el vertical, los agrupamientos pueden ser explicados porque el producto de una empresa sirve como insumo de otra, de acuerdo con un encadenamiento productivo. Backstrom y Karlsson (1999) y Howells (1999) afirman que el agrupamiento no sólo se refiere a empresas o industrias, sino que considera instituciones de educación superior o agencias públicas o privadas de servicios legales o administrativos.

Para el caso de una región económica como la conformada por las economías de Baja California y California, donde se da una fuerte interacción en los mercados laborales y de insumos de las empresas norteamericanas, asiáticas y mexicanas, la perspectiva de afinidad parece muy adecuada para explicar la fuerza de la relación regional y binacional que se desarrolla entre la homogeneidad de intereses locales y la heterogeneidad de las diferentes redes empresariales (Westlund, 1999). Los niveles de especialización industrial de Baja California darían lugar a la idea de que las empresas con mayor poder de mercado no tratan de expulsar a otras con menor poder para optimizar sus funciones de producción y de beneficio, no sólo porque sus mercados son diferentes, sino por la incertidumbre de poder emplear en el largo plazo la capacidad instalada plenamente. Por lo tanto, buscarían controlar los nichos de demanda final y subcontratar, primero, a sus proveedores de San Diego y luego a los que fuera posible reclutar entre las micro y pequeñas empresas (Mypes) locales con criterios de calidad para evitar inversiones riesgosas (Mungaray, 1997). En esta lógica se realiza el ajuste regional de los salarios en la manufactura, ya que al ser desplazados los procesos intensivos en trabajo hacia la frontera norte mexicana por parte de las empresas transnacionales, éstas a su vez se especializan en procesos de alta tecnología, investigación y desarrollo del lado de Estados Unidos. Por tanto, la especialización industrial promueve la calificación de la fuerza de trabajo que se incrementa en ambos lados de la frontera, lo que hace aumentar la demanda de trabajo calificado a la vez que sus respectivas remuneraciones (Feenstra y Gordon, 1995). Se esperaría una tendencia general 
al incremento de la demanda de personal capacitado de la mano del desarrollo, como resultado tanto de la acumulación de capital como del cambio tecnológico (Mincer, 1995). Al incrementarse la proporción de ingresos por persona es probable que los niveles educativos y los salarios relativos del personal capacitado aumenten (Meza, 1999). Si bien es cierto que el acervo de capital humano influye positivamente el crecimiento económico y principalmente al capital físico al ser ambos complementarios, también lo es que mantiene influencia directa sobre el ingreso per cápita (Barro, 1994).

En el caso de la región fronteriza de Baja California, el objetivo de la subcontratación en las empresas transnacionales (en su mayoría japonesas y estadounidenses) es el aprovechamiento de las ventajas que ofrece la región respecto a localización, especialización, calificación de la mano de obra, externalidades y diferenciales salariales. La importancia de la educación, tanto por su calidad y disponibilidad, ha permitido mantener una estructura productiva de costos laborales bajos y una fuerza de trabajo con gran capacidad de aprendizaje y alta productividad. La creación de esta ventaja competitiva ha impulsado el asentamiento y la consolidación productiva de empresas internacionales con diversos niveles de desarrollo tecnológico. La complementariedad de estos factores estimula el desarrollo de proveedores de insumos industriales en el lado de California y con ello el crecimiento industrial y de los servicios profesionales en el área de San Diego y sur de California (Mungaray et al., 2001).

Las diferencias en vocaciones industriales manufactureras, en aspectos educativos y experiencia laboral en las ciudades fronterizas del norte de México, dan a conocer que la apertura económica ha afectado la dispersión salarial debido a los diferenciados efectos de la experiencia laboral y de la educación. Para Carrillo y Hualde (1998), en las ciudades fronterizas de Baja California algunas plantas industriales se han especializado en fases concretas de procesos industriales que consideran mayor calificación de la mano de obra, lo que influye fuertemente en el crecimiento de la producción. El entorno de apertura económica propiciado por el Tratado de Libre Comercio de América del Norte (TLCAN) ha fortalecido el proceso de integración económica regional. Ello ha impulsado mayores movimientos en los flujos de in- 
versión extranjera directa y de bienes y servicios en ambos lados de la frontera norte. En este escenario la fortaleza económica de Baja California ha radicado en el desarrollo de su condición de frontera con la economía más grande del mundo. A su natural disponibilidad de capital humano, se han agregado los flujos migratorios de otras entidades del país en busca de una mejor oportunidad de empleo. La idea de acumular una mayor dotación de capital humano contribuye a un mejoramiento de la productividad, lo cual puede incrementar el valor agregado en las actividades económicas, con efectos favorables en los ingresos salariales y el bienestar.

\section{Metodología}

El propósito de este trabajo es analizar si variables proxy de capital humano como el grado de escolaridad, la experiencia laboral y la especialización del personal ocupado han sido relevantes en la determinación de los ingresos promedios de los trabajadores de la industria manufacturera de las ciudades de Tijuana y Mexicali, Baja California (Cabrera, 2007). Para ello se estima una ecuación de ingresos semilogarítmica minceriana (Mincer, 1974) de la siguiente forma:

$$
Y / L_{i t}=\delta_{0}+\delta_{1} A E_{i t}+\delta_{2} E X_{i t}+\delta_{3} E X^{2}{ }_{i t}+\delta_{4} E S_{i t}+\lambda \sum d_{i}+\varphi \sum d_{t}+\varepsilon_{i j}
$$

donde

$\mathrm{Y} / \mathrm{L}_{\mathrm{it}}=$ ingresos promedios del trabajador manufacturero

$\mathrm{AE}_{\mathrm{it}}=$ años de escolaridad formal. ${ }^{1}$

$\mathrm{EX}_{\mathrm{it}}=$ años de experiencia.

$\mathrm{EX}_{\mathrm{it}}{ }^{2}=$ años de experiencia al cuadrado.

$\mathrm{ES}_{\mathrm{it}}=$ índice de especialización del personal ocupado.

1 AE, se calculó considerando los años de instrucción formal, EX como [edad-escolaridad-6] y la especialización de la fuerza de trabajo con base en $I E\left(x_{i r}=\left[\left(x_{i r} / x_{r}\right) /\left(x_{i n} / x_{n}\right)\right]\right.$ donde $x$ es el personal ocupado, $i$ es la rama económica, $r$, la ciudad y $n$ el estado. 
El subíndice $i$ denota la rama económica y $t$ el horizonte temporal. La ecuación está fundamentada en la teoría del capital humano y de acuerdo con Zepeda y Ghiara (1999) representa un intento pragmático que modela la relación funcional del ciclo de vida de los ingresos, la educación y el entrenamiento. Específicamente, $d_{i}$ y $d_{t}$ son matrices que agrupan vectores de variables dummy que capturan en el modelo el efecto de los cortes trasversales (i-ramas) y periodos de tiempo ( $t$ años) en la ecuación de regresión. Cabe precisar que los estimadores $\lambda$ у $\varphi$ agrupan los parámetros asociados a los efectos fijos de cada industria y cada periodo de tiempo, pues la estimación que se realiza corresponde a una estructura de datos en panel.

La hipótesis que se evalúa con el estadístico $F$ es $\left\{\mathrm{H}_{\mathrm{o}}: \alpha_{\mathrm{i}}=\alpha\right\}$, que establece que el modelo tiene un intercepto común para todas las ramas analizadas. El término (n-1, nT-n-k) denota los grados de libertad en el numerador y denominador y el contraste se efectúa considerando un nivel de 95\% de significancia estadística. De acuerdo con Wooldridge (2001), la aceptación de esta hipótesis descarta una estimación con efectos fijos, lo que significa que se procede únicamente a estimar una regresión agrupada con NT observaciones en donde sólo se incorporan a la ecuación de regresión las variables de capital humano y no los vectores de variables dicotómicas. Tanto para Tijuana como para Mexicali se plantean tres versiones distintas de un modelo con efectos individuales de la forma:

$$
y_{i t}=x_{i t}^{\prime} \beta+\eta_{i}+v_{i t} \quad(\mathrm{i}=1, \ldots . . \mathrm{N} ; \mathrm{t}=1, \ldots . . \mathrm{T})
$$

En donde $x_{i t}$ es un vector $\mathrm{k}^{*} 1$ de variables explicativas, $\beta$ es el vector de parámetros a estimar, $\eta_{i}$ es un efecto individual y $v_{i t}$ un termino de perturbación. Como bien apuntan Arrellano y Bover (1990), si el modelo incluye efectos temporales, se suponen incluidos en $\beta$ en cuyo caso $x_{i t}$ contiene las correspondientes variables ficticias en el tiempo. En el modelo de efectos fijos, los $\eta_{i}$ son tratados como un conjunto de $\mathrm{N}$ coeficientes adicionales que se pueden estimar junto con $\beta$. En el caso del modelo de efectos aleatorios ${ }^{2}$ se parte de que $\eta_{i}$ es una varia-

${ }^{2}$ No se estiman efectos aleatorios para probar $H_{0}: \sigma_{\mu}^{2}=0$ en virtud de que los resultados son menos robustos. 
ble aleatoria inobservable independiente de $x_{i t}$ que forma parte del término de perturbación compuesto: ${ }^{3}$

$$
\mu_{i t}=\eta_{i}+v_{i t}
$$

Los tres modelos que se estiman son los siguientes. ${ }^{4}$

$$
\begin{aligned}
& Y / L_{i t}=\delta_{1} A E_{i t}+\delta_{2} E X_{i t}+\delta_{3} E X^{2}{ }_{i t}+\delta_{4} E S_{i t}+\lambda \sum d_{i}+\varphi \sum d_{t}+\varepsilon_{i j} \\
& Y / L_{i t}=\delta_{1} A E_{i t}+\delta_{2} E X_{i t}+\delta_{3} E X^{2}{ }_{i t}+\delta_{4} E S_{i t}+\lambda \sum d_{i}+\varepsilon_{i j} \\
& Y / L_{i t}=\delta_{1} A E_{i t}+\delta_{2} E X_{i t}+\delta_{3} E X^{2}{ }_{i t}+\delta_{4} E S_{i t}+\varphi \sum d_{t}+\varepsilon_{i j}
\end{aligned}
$$

La elaboración de la base de datos sobre la industria manufacturera de Tijuana y Mexicali se realizó integrando información por ciudad, de acuerdo a la clasificación por áreas metropolitanas obtenida de la Encuesta Nacional de Empleo Urbano (ENEU) del Instituto Nacional de Estadística, Geografía e Informática (INEGI). En la integración de los datos se consideraron para Tijuana 23 ramas y para Mexicali 28. En el primer caso el periodo de estudio comprende desde el primer trimestre de 1994 hasta el último trimestre de 2001, y para Mexicali desde el primer trimestre de 1998 hasta el cuarto trimestre de 2001. Los datos de ingresos se convirtieron de precios corrientes a precios constantes. Cabe precisar que en las estimaciones se utilizaron promedios trimestrales para disponer de una base de datos con frecuencia anual. La razón fundamental por la cual se integra información agregada es porque ayudó a mejorar el ajuste de las estimaciones así como estadísticos de prueba como la t y F. La muestra para Tijuana es de 184 observaciones y para Mexicali de 112. Por otra parte, conviene insistir en que el modelo que se estima corresponde a una especifica-

\footnotetext{
${ }^{3}$ Por ello es común que a éstos modelos se les conozca en la literatura como modelos con errores compuestos (error components).

${ }^{4} \mathrm{Al}$ introducir las variables binarias, se omite el intercepto para evitar el problema de la trampa de la variable dicótoma que deriva en una situación de colinealidad perfecta o una relación exacta entre las variables.
} 
ción no extendida a una desagregación de la escolaridad por niveles. Esto en razón de que los datos de la Encuesta Nacional de Empleo Urbano en su versión impresa, no reportan tabulados que permitan obtener cifras referentes a los folios de los jefes del hogar. La disponibilidad de este tipo de información va más allá de los alcances de esta investigación, no obstante se visualiza como un esfuerzo de investigación posterior. La idea de contemplar el periodo de estudio 1994-2001 obedece a la necesidad de construir una primera base de datos de un periodo mucho más largo que a futuro se está considerando integrar con información tanto de la Encuesta Nacional de Empleo Urbano (ENEU) como de la Encuesta Nacional de Empleo (ENE) y la Encuesta Nacional de Ocupación y Empleo (ENOE). Cabe agregar que la información de los ingresos está relacionada únicamente con el segmento de trabajadores asalariados y la experiencia se calculó de acuerdo con lo establecido en la nota número 1.

\section{Análisis de resultados}

La información sobre los ingresos de los trabajadores de Tijuana indica una tendencia creciente a la par con el número de años de educación. Para 1998-1, los trabajadores con un nivel educativo de 0 a 6 años (primaria) y de 7 a 9 años (secundaria) tuvieron ingresos promedio de 2,655 y 2,675 pesos respectivamente. Los trabajadores con preparatoria y carreras técnicas (10 a 12 años) obtuvieron los mayores ingresos promedio con 2,748 pesos pero con una gran dispersión salarial reflejada en una desviación estándar de 4,375 pesos. Los trabajadores con licenciatura y más (13 años y más) presentaron menores ingresos a los anteriores. Para 1999-4 los trabajadores que más ingresos tuvieron fueron los de licenciatura y en 2001-4 los trabajadores con licenciatura y más despuntan con mayores ingresos y amplia diferencia respecto al nivel preparatoria, con ingresos promedio de 5,492 pesos. En Mexicali, para 1998-1 los trabajadores que más ingresos obtuvieron fueron los de nivel preparatoria, con 4,047 pesos y una amplia dispersión salarial. Para 1999-4, de nuevo los trabajadores de nivel preparatoria obtuvieron los mayores ingresos con 5,491 pesos, si bien para 2001-4 los trabajadores de nivel licenciatura tuvieron las mejores re- 
muneraciones promedio. En ambos municipios destaca que es hasta el cuarto trimestre del 2001 cuando los trabajadores de nivel licenciatura y más logran los mayores ingresos, lo cual es acorde con el mayor número de años de educación. La dispersión salarial de mayor a menor indica una evolución de mayor diversidad a más homogeneidad en los empleos y remuneraciones para este grupo de trabajadores en el sector manufacturero, lo que en la estructura del mercado laboral refleja la movilidad de la fuerza de trabajo al pasar de unos sectores a otros.

En 1998-1 las industrias de alimentos y bebidas, de la madera, maquinaria y productos metálicos de Tijuana, experimentaron los mayores ingresos medios laborales con 2,823, 2,790 y 2,810 pesos, respectivamente. Para 1999-4, industrias de la madera, productos minerales no metálicos y productos metálicos obtuvieron los mayores ingresos con 3,883, 3,641 y 3,792 pesos, respectivamente, y en el 2001-4 destacan alimentos y bebidas, de la madera e imprentas y editoriales con 4,231, 4,106 y 3,965 pesos respectivamente. En Mexicali, para 1998-1 sobresalen las industrias de la madera, productos minerales no metálicos y productos metálicos con ingresos de 3,129, 3,317 y 5,006 pesos respectivamente. Para 1999-4, destacan la madera, productos minerales no metálicos y maquinaria y productos metálicos con ingresos de 4,292, 4,583 y 3,813 pesos, respectivamente. Para 2001, las industrias de la madera y alimentos y bebidas reciben los mayores ingresos. En suma, la mayoría de las industrias con mayores ingresos coinciden con mayores niveles de años de educación en la manufactura para ambos municipios.

Para el conjunto de los trabajadores manufactureros, en 2001-4 los ingresos medios más altos y mayores niveles de educación correspondieron a Mexicali con 4,819 pesos y 8.7 años, frente a Tijuana con 4,761 pesos y 7.4 años respectivamente.

De acuerdo con los censos de población de INEGI (2000), se observa cómo Mexicali mantiene un nivel de escolaridad mayor de su población en comparación con Tijuana, tanto en secundaria como en profesional, con $64.8 \%$ contra $61.3 \%$ y $1.6 \%$ contra $0.9 \%$, respectivamente.

Si bien el entorno del TLCAN y la creciente entrada de inversión extranjera directa (IED) a Baja California favorecen la economía y el 
Cuadro 1. Ingresos, educación y experiencia en el sector manufacturero en Mexicali y Tijuana en 2001-04

\begin{tabular}{lcccc}
\hline Municipal & Edad & $\begin{array}{c}\text { Ingreso } \\
\text { mensual* }\end{array}$ & $\begin{array}{c}\text { Años de } \\
\text { estudio }\end{array}$ & $\begin{array}{c}\text { Años de } \\
\text { experiencia }\end{array}$ \\
\hline $\begin{array}{l}\text { Mexicali } \\
\text { Promedio }\end{array}$ & 32.4 & 4819.6 & 8.7 & 17.9 \\
$\begin{array}{l}\text { Desv. estándar } \\
\text { Tijuana }\end{array}$ & 9.9 & 2026.2 & 3.0 & 11.0 \\
$\begin{array}{l}\text { Promedio } \\
\text { Desv. estándar }\end{array}$ & 33.1 & 4761.6 & 7.4 & 19.4 \\
\hline
\end{tabular}

Fuente: Elaboración propia con datos de la Encuesta Nacional de Empleo Urbano, ENEU, 2001-04, INEGI. * Pesos corrientes.

Cuadro 2. Niveles de escolaridad de la población total (2000)

\begin{tabular}{lccc}
\hline Municipio & Primaria & Secundaria & Profesional \\
\hline Tijuana & $89.9 \%$ & $61.3 \%$ & $0.9 \%$ \\
Mexicali & 89.7 & $64.8 \%$ & $1.6 \%$ \\
\hline
\end{tabular}

Fuente: Elaboración propia con datos de los censos de población, INEGI.

empleo en Mexicali y Tijuana desde 1994, también el crecimiento demográfico y los movimientos de población en las ciudades fronterizas de México y Estados Unidos han favorecido el desarrollo de mercados regionales de bienes y servicios en ambos lados de la frontera, impulsando la demanda de trabajo relativamente calificado. Para 2001, tanto para Mexicali como para Tijuana se experimentaron mayores índices de especialización en la industria electrónica, productos de madera, vestido, plástico y estructuras metálicas. Esto es indicador de la vocación industrial y fuente de empleos y bienestar en la región fronteriza de Baja California. Por otra parte, las industrias que registraron niveles de especialización más bajos en Mexicali fueron la industria de preparados de frutas y legumbres, otras industrias textiles, maquinaria, equipos y aparatos eléctricos con ingresos promedio por hora de 
18.9, 18.7 y 19.3 pesos. En el caso de Tijuana, las industrias menos especializadas son: productos alimenticios, industria del hierro y del acero, y equipo de transporte con participaciones e ingresos de 20.9, 18.8 y 20.1 pesos por hora respectivamente.

El modelo [1.1] es una versión no restringida en donde los coeficientes de las pendientes son constantes pero la intersección varía conforme las ramas económicas y el tiempo. Por su parte, el modelo [1.2] se caracteriza porque los coeficientes de las pendientes son también constantes pero la intersección varía para cada rama económica. En este caso particular, aun cuando la intersección varía para cada rama económica, cada intersección es invariante en el tiempo y recoge el efecto individual de cada rama. La especificación [1.3] permite capturar además de las variables de capital humano el efecto del tiempo en la función de ingresos promedios. La prueba F para el caso de Tijuana que aísla los efectos de las ramas económicas de los efectos de los periodos de tiempo, arroja un valor calculado de 5.01 que excede el valor crítico de 2.6, determinando que hay efectos diferenciados en las industrias analizadas sobre los ingresos promedio. En el caso de Mexicali también se obtiene un resultado en la misma dirección, pues el valor calculado de 3.01 es mayor al valor crítico. Es importante anotar que a reserva de estos resultados, se estiman las tres versiones especificadas con el interés de evaluar lo resultados de forma comparativa (véanse los cuadros 3, 4 y 5).

Las distintas formas funcionales corresponden a una ecuación semilogarítmica tipo Mincer (1974). Esto significa que los coeficientes obtenidos de las variables explicativas $\mathrm{AE}_{\text {it }}, \mathrm{EX}_{\mathrm{it}} \mathrm{y} \mathrm{AE}^{2}{ }_{\text {it }}$, representan semielasticidades. Un aspecto importante es que la epecialización del personal ocupado $\mathrm{ES}_{\mathrm{ir}}$ resultó ser una variable no significativa y por ello no se incorpora en ningún modelo. Por otro lado, cabe señalar que las estimaciones se efectuaron utilizando el método de White que produce errores estándar robustos ante la presencia de heterocedasticidad. ${ }^{5}$

${ }^{5}$ El método implica que para cualquier coeficiente de regresión parcial $\beta_{j^{\prime}}$ $\operatorname{var}\left(\hat{\beta}_{j}\right)=\frac{\sum \hat{\omega}_{j i} \hat{\mu}_{i}^{2}}{\left(\sum \hat{\omega}_{i j}^{2}\right)^{2}}$, donde $\hat{\mu}_{i}$ son los residuos obtenidos de la regresión original y $\hat{\omega}_{j}$ son los residuos proporcionados por la regresión auxiliar de $\mathrm{X}_{j}$ sobre las regresoras restantes. 
Cuadro 3. Resultados de estimación del modelo [1.1]

\begin{tabular}{|c|c|c|c|c|}
\hline & \multicolumn{2}{|c|}{$\begin{array}{c}\text { Tijuana } \\
\text { 1994T1-2001T4 }\end{array}$} & \multicolumn{2}{|c|}{$\begin{array}{c}\text { Mexicali } \\
\text { 1998T1-2001T4 }\end{array}$} \\
\hline & Coeficiente & t-estadístico & Coeficiente & t-estadístico \\
\hline $\mathrm{AE}_{\mathrm{it}}$ & 0.16 & 3.27 & 0.20 & 5.43 \\
\hline$E X_{i t}^{11}$ & 0.14 & 3.00 & 0.10 & 3.08 \\
\hline $\mathrm{EX}^{2}{ }_{\text {it }}$ & -0.00 & -1.81 & -0.00 & -1.82 \\
\hline $\mathrm{R}^{2}$ ajustado & \multicolumn{2}{|c|}{0.58} & \multicolumn{2}{|c|}{0.55} \\
\hline Tijuana NT & \multicolumn{2}{|c|}{184} & \multicolumn{2}{|c|}{112} \\
\hline
\end{tabular}

Cuadro 4. Resultados de estimación del modelo [1.2]

\begin{tabular}{|c|c|c|c|c|}
\hline & \multicolumn{2}{|c|}{$\begin{array}{c}\text { Tijuana } \\
\text { 1994T1-2001T4 }\end{array}$} & \multicolumn{2}{|c|}{$\begin{array}{c}\text { Mexicali } \\
\text { 1998T1-2001T4 }\end{array}$} \\
\hline & Coeficiente & t-estadístico & Coeficiente & t-estadístico \\
\hline $\mathrm{AE}_{\mathrm{it}}$ & 0.03 & 0.65 & 0.18 & 4.99 \\
\hline$E X_{i t}^{1 t}$ & 0.27 & 5.92 & 0.11 & 3.34 \\
\hline $\mathrm{EX}^{2}{ }_{\text {it }}$ & -0.01 & -3.91 & 0.00 & -1.94 \\
\hline $\mathrm{R}^{2}$ ajustado & \multicolumn{2}{|c|}{0.34} & \multicolumn{2}{|c|}{0.52} \\
\hline Tijuana NT & \multicolumn{2}{|c|}{184} & \multicolumn{2}{|c|}{112} \\
\hline
\end{tabular}

Cuadro 5. Resultados de estimación del modelo [1.3]

\begin{tabular}{|c|c|c|c|c|}
\hline & \multicolumn{2}{|c|}{$\begin{array}{c}\text { Tijuana } \\
\text { 1994T1-2001T4 }\end{array}$} & \multicolumn{2}{|c|}{$\begin{array}{c}\text { Mexicali } \\
\text { 1998T1-2001T4 }\end{array}$} \\
\hline & Coeficiente & t-estadístico & Coeficiente & t-estadístico \\
\hline $\mathrm{AE}_{\mathrm{it}}$ & 0.19 & 6.93 & 0.25 & 10.95 \\
\hline$E X_{i t}^{1 t}$ & 0.12 & 4.23 & 0.07 & 2.97 \\
\hline $\mathrm{EX}^{2}{ }_{\text {it }}^{1 \mathrm{i}}$ & 0.00 & -2.26 & -0.01 & -1.10 \\
\hline $\mathrm{R}^{2}$ ajustado & \multicolumn{2}{|c|}{0.67} & \multicolumn{2}{|c|}{0.34} \\
\hline Tijuana NT & \multicolumn{2}{|c|}{184} & \multicolumn{2}{|c|}{112} \\
\hline
\end{tabular}


En lo que respecta al caso de Tijuana, los resultados indican que el modelo [1.3] es el que mejor ajuste produce, pues se puede constatar que las tres variables de capital humano son estadísticamente significativas. Ello contrasta con el modelo [1.2], en donde los años de escolaridad no son determinantes de los ingresos. El efecto tiempo introducido en [1.3] y que se captura a través de $\sum_{t}^{8} d_{t}$ sugiere que la función de ingresos puede desplazarse en el tiempo, pues los coeficientes asociados a las variables dicotómicas así lo confirman.

Considerando esta última especificación, es claro que los años de escolaridad son más importantes que la experiencia laboral, aun cuando ésta también es muy significativa. Hay que advertir que habrá ramas en donde los niveles de ingreso de los trabajadores se ubican por arriba o por debajo de la media del conjunto de las 23 ramas consideradas, por lo que el impacto individual pudiera variar. En el caso de Tijuana se puede decir que los resultados relacionados con la escolaridad y la experiencia laboral son consistentes con el tipo de relación esperada.

Para Mexicali también se descarta la estimación de un modelo

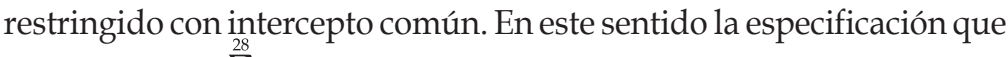
sólo incorpora $\sum_{i=1}^{28} d_{i}$ y omite los efectos temporales, es el modelo que mejor resultados arroja en cuanto a significancia estadística. Al igual que en el caso de Tijuana, se comprueba que la variable que más peso tiene en la determinación de los ingresos promedio es la escolaridad y en menor medida la experiencia laboral. En ambos casos la relación observada es positiva y en lo referente a la experiencia laboral al cuadrado el signo es negativo tal y como se esperaría. Si se considera que el modelo de panel más robusto para Tijuana contempla $\sum_{t=1}^{8} d_{t}$ y para Mexicali $\sum_{t=1}^{8} d_{t}$, entonces se puede argumentar razonablemente que en este último caso, las características particulares de cada rama son relevantes en la determinación de los ingresos promedio por los efectos fijos que tienen.

\section{Conclusiones}

A partir de los resultados obtenidos se puede concluir que la especialización del personal ocupado no es una variable relevante en la de- 
terminación del ingreso promedio de los trabajadores de la industria manufacturera de Tijuana y Mexicali. Si bien es cierto que la especialización constituye una variable representativa de las economías de aglomeración de naturaleza intraindustrial que pueden desencadenar efectos favorables sobre los niveles de empleo en los mercados laborales locales, también lo es que está estrechamente relacionada con los procesos de innovación y dotación de capital humano. No es fortuito que en las especificaciones econométricas no sea significativa, pues en las ecuaciones de ingreso tipo minceriana normalmente no figura explícitamente como variable independiente.

El hecho de que la escolaridad sea la variable de capital humano más importante en las estimaciones realizadas para Tijuana, significa que en el mercado de trabajo local el nivel de educación se convierte en un criterio fundamental de la negociación salarial. También puede estar indicando que es un mercado de trabajo especializado en donde la escolaridad se asocia con un desarrollo de habilidades, destrezas y capacidades de la fuerza de trabajo. En el caso de Mexicali se presenta el mismo fenómeno, aun cuando el número de ramas estudiadas es mayor que las consideradas en Tijuana.

La estrecha relación positiva entre ingresos y años de escolaridad supone una elevada exigencia del mercado de trabajo local en materia de educación, que plantea la necesidad de fortalecer constantemente el vínculo entre los centros de producción y de educación básica, media y superior, con el propósito de adecuar los procesos de enseñanza-aprendizaje a las necesidades que plantea el desarrollo empresarial de ambas ciudades fronterizas. La implicación de política educativa que se deriva del trabajo empírico, es que en lo que respecta a la educación media técnica y superior, se deben adoptar medidas institucionales que garanticen un adecuado diseño y reestructuración de los programas de estudio y sus perfiles de egreso, de forma que las competencias técnicas y profesionales estén ligadas con la visión empresarial de largo plazo.

Los resultados sugieren que la experiencia laboral también es una variable importante en la determinación de los ingresos. Se entiende que al representar habilidades y capacidades acumuladas adquiridas en los distintos centros de trabajo, contribuye a eficientizar el proceso 
de producción y con ello a elevar los niveles de productividad laboral. En este sentido el vínculo positivo entre experiencia laboral e ingresos puede significar que las remuneraciones estén ajustadas a criterios de productividad y eficiencia, en cuyo caso predominaría un esquema de negociación salarial bilateral sobre uno de negociación colectiva. No obstante, hay que advertir que esto dependería de las características particulares de cada rama o industria, de donde se concluye que las ramas con mayor desarrollo tecnológico absorberían fuerza de trabajo más especializada, con altos niveles de escolaridad y basta experiencia laboral.

\section{Bibliografía}

Arellano, M. y O. Bover (1990), "La econometría de datos de panel", Investigaciones Económicas, vol. XIV, núm. 1, pp. 5-44.

Alegría, O. Tito (1992), Desarrollo urbano en la frontera México-US, Méxi$\mathrm{co}$, Conaculta.

Ampudia R., Lourdes (2007), “Análisis del mercado de trabajo: comportamiento salarial y su rendimiento de acuerdo al nivel educativo en Ciudad Juárez 1987-1998", Aportes, año XII, núm. 35, mayo-agosto, pp. 117-130.

Barro, Robert (1994), "Economic Growth in a Cross Section of Countries", Quarterly Journal of Economics, vol. CVI, pp. 363-394. Backstrom, M. y C. Karlsson (1999), Specialisation and Clustering in an Industrial District. The Case of the County of Jonkoping, Jonkoping International Business School, documento de trabajo.

Barceinas P., Fernando (2001), Capital humano y rendimientos de la educación en México, tesis doctoral, Barcelona, España, Universidad Autónoma de Barcelona.

Burgos, B. (2005), Efectos de la apertura externa sobre el mercado laboral de la región noroeste de México, tesis de doctorado en Ciencias Económicas, Tijuana, UABC.

Cabrera, C. (2007), Especialización industrial, calificación laboral e ingresos del trabajo en la manufactura en Baja California, tesis de doctorado en Ciencias Económicas, Tijuana, UABC. 
Carrillo, J. y A. Hualde (1998), “Third Generation Maquiladoras? The Delphi-General Motors Case", Journal of Borderlands Studies, vol. XIII, núm. 1.

Cortez, W. W. (2005), “Dispersión y estabilidad de las diferencias salariales interestatales en México, 1984-2000", Investigación Económica, vol. LXIV, núm. 253, jul-sep, pp. 123-158.

Feenstra, R. C. y H. H. Gordon (1995), Foreign investment, Outsourcing and Relative Wages, documento de trabajo del NBER, núm. 5121.

Hueca Reynos, Luis (1994), "La rentabilidad de la escolaridad en los hogares asalariados de México durante 1984-2000", Problemas del Desarrollo, vol. 35, núm. 138, julio-septiembre, pp. 125-154.

Howells, J. (1999), "Regional Systems of Innovation", en D. Archibugi, J. Howells y J. Michie (eds.), Innovation Policy in a Global Economy, Cambridge University Press.

INEGI (1994 y 1999), Censos Económicos de Baja California, México.

Mendoza, J. E. (2002), “Educación, experiencia y especialización manufacturera en la frontera norte de México", Comercio Exterior, vol. 52 (4), pp. 300-308.

Meza, L. (1999), "Cambios en la estructura salarial de México en el periodo 1988-1993 y el aumento en el rendimiento de la educación superior", El Trimestre Económico, vol. LXVI (2), núm. 262, abril-junio, pp. 189-226.

Mincer, J. (1974), Schooling Experience and Earnings, Nueva York, NBER. (1995), "Economic Development, Growth of Human Capital, and the Dynamics of the Wage Structure", Journal of Economic Growth, núm. 1, pp. 29-48.

Mungaray, A. (1997), Organización industrial de redes de subcontratación para pequeñas empresas en la frontera norte de México, México, NAFIN. (2001), "Especialización económica y promoción empresarial en Baja California”, El Mercado de Valores, vol. 11 (10), pp. 1-27. P. Moctezuma y R. Varela (2001), "Higher Education and Economic Growth in the California-Baja California Region", en J.W. Wilkie, E. Alemán y J.G. Ortega (eds.), Statistical Abstract of Latin America, vol. 37, pp. 1035-1043. 
Palacio, J. I. y J. H. Simón (2002), “Dispersión salarial y negociación colectiva en España", Cuadernos de Relaciones Laborales, vol. 20, núm 1. pp. 169-187. (2004), “Dispersión salarial entre establecimientos y desiguladad salarial”, Revista de Economía Aplicada, vol. XII, núm 36, pp. 4781.

Urciaga, J. (2002), “Los rendimientos privados de la escolaridad formal en México", Comercio Exterior, vol. 52 (4), pp. 324-330.

Villavicencio, D. (2002), "La gestión del conocimiento productivo: las normas ISO y los sistemas de aseguramiento de calidad", Comercio Exterior, vol. 52 (6), pp. 508-520.

Westlund, H. (1999), “An Interaction-cost Perspective on Networks and Territory", Annals of Regional Science, núm. 33, pp. 93-121.

Wooldridge, M. J. (2001), Econometric Analysis of Cross Section and Panel Data, MIT Press.

Zepeda, M. E. y R. Ghiara (1999), “Determinación del salario y capital humano en México: 1987-1993", Economía, Sociedad y Territorio, vol. II (5), pp. 67-116.

Recibido en febrero de 2008 Segunda versión recibida en junio de 2008 Aprobado en septiembre de 2008 